\title{
Notícias e mudanças no Journal of Epilepsy and Clinical Neurophysiology
}

\footnotetext{
$\mathrm{O}$

Dr. Luciano de Paola assume como novo Editor do JECN. O Dr. Luciano é bastante conhecido na comunidade da neurofisiologia clínica e epileptologia brasileira e dispensa maiores apresentações. Acreditamos que com suas características de bom senso e entusiasmo nosso jornal ganhará novo impulso, além de manter a qualidade e periodicidade dos artigos originais e de revisão.

A Dra. Magda Lahorgue Nunes, Presidente da Liga Brasileira de Epilepsia no biênio 2004-2006, re-assume o cargo de Editor Executivo do jornal. Nossos agradecimentos ao Dr. Jaderson Costa da Costa pelo apoio e dedicação como Editor Executivo durante mais um período que se encerra.

O quadro de Editores Associados também foi renovado com a entrada de dois jovens pesquisadores: os Drs. Lauro Wichert Ana da FMUSP-Ribeirão Preto e Fulvio Alexandre Scorza da Neurologia Experimental da UNIFESP, que se somam aos Drs. André Palmini e Elza Márcia Yacubian.

Finalmente, é com grande satisfação que informo aos leitores que o JECN já se encontra disponível na SciELO (http://www.scielo.br). A Scientific Electronic Library Online (SciELO) é uma biblioteca eletrônica que abrange uma coleção selecionada de periódicos científicos brasileiros. A SciELO é o resultado de um projeto de pesquisa da FAPESP - Fundação de Amparo à Pesquisa do Estado de São Paulo, em parceria com a BIREME - Centro Latino-Americano e do Caribe de Informação em Ciências da Saúde. A partir de 2002, o Projeto conta com o apoio do CNPq Conselho Nacional de Desenvolvimento Científico e Tecnológico. A interface SciELO proporciona acesso à sua coleção de periódicos através de uma lista alfabética de títulos, ou por meio de uma lista de assuntos, ou ainda através de um módulo de pesquisa de títulos dos periódicos, por assunto, pelos nomes das instituições publicadoras e pelo local de publicação. Portanto, os artigos publicados no JECN passam a ter uma maior "visibilidade" e podem ser obtidos na íntegra em qualquer parte do mundo.
}

Fernando Cendes 